\title{
BANDWIDTH AWARE MULTIPATH GEOGRAPHIC ROUTING PROTOCOL (BAMGRP) for RELIABLE VIDEO TRANSMISSION in MULTIMEDIA VEHICULAR AD HOC NETWORKS
}

\author{
${ }^{1}$ R.Tamilselvi ${ }^{2}$ Dr.V.Kathiresan \\ ${ }^{1}$ Department of Computer Science, Dr.SNS Rajalakshmi college of Arts \& Science, \\ Coimbatore - 641 049, Tamil Nadu, India. \\ ${ }^{2}$ Department of Computer Applications(PG), Dr.SNS Rajalakshmi college of Arts \& Science, \\ Coimbatore - 641 049, Tamil Nadu, India. \\ ${ }^{1}$ rtamilu@gmail.com \\ 2 vkathirmca@gmail.com
}

\begin{abstract}
Reliable video transmission in VANET is a challenging task. Multimedia VANETs are wellsuited for capturing and sharing environmental monitoring, surveillance, traffic accidents, and disasterbased video smart city applications. This article aims in proposing Bandwidth Aware Multipath Geographic Routing Protocol (BAMGRP) for Reliable Video Transmission in Multimedia Vehicular Ad hoc Networks. Multiple paths are constructed using the AOMDV routing strategy. Time-slots are allocated for the video transmission request(s). Available bandwidth is obtained for the available paths. Then minimum predicted available bandwidth is measured. The concurrent video transmission requests are sorted based on their bandwidth demand in a descending order. Then bandwidth is allocated for video transmission. Simulations are performed using MATLAB. Results prove that the proposed BAMGRP performs better in terms of route length, packet end-to-end delay, freezing delay, number of delivered packets and packet loss ratio.
\end{abstract}

Keywords: Multimedia VANET, MANET, Video Transmission, Bandwidth, route length, packet end-to-end delay, freezing delay, number of delivered packets, packet loss ratio, reliability.

\section{INTRODUCTION}

An ad hoc network is an autonomous system of wireless nodes that considerately structure a network devoid of any explicit management [5]. Every node in an ad hoc network is in charge of routing information among its neighbors. When nodes are free to move randomly and organize themselves arbitrarily, it is referred as Mobile Ad Hoc NETworks (MANETs) [6]. As an evolution of traditional MANETs, VANETs (Vehicular Ad hoc NETworks) [7] include communications between vehicles (Vehicle to Vehicle, also known as V2V communications) on the roads and with the road communication infrastructure (Vehicle to Infrastructure, also known as V2I communications). Vehicular ad hoc networks (VANETs) are temporal, selforganized networks where vehicles send and receive information to other vehicles (V2V communications) or to fixed infrastructure points (V2I communications). These communications might enable a high number of potential applications [4]. Vehicular Ad Hoc Networks (VANETs) are focused to foster Information and Communication Technology to perk up and alleviate transportation problems. Vehicles can work together between themselves to circulate not only trouble-free scalar data, e.g., text messages, but also live multimedia content, e.g., videos.

Such scenario motivated industries and manufacturers to endow with drivers and passengers with a wide scope of novel real-time multimedia services, ranging from safety and security traffic warnings to live entertainment and advertising video flows [1]. Among several Information and Communication Technologies (ICT) services, video surveillance is the key service for developing smart city scenarios which obtains an imperative notice from governments, car manufacturers, academia, and society [1]. At the present time, the allocation of real-time multimedia content over Vehicular Ad-Hoc Networks (VANETs) is flattering a realism and consenting to drivers / passengers for experiencing with on-road videos in smart cities [2,3]. Multipath routing protocols even for energy efficient data transmission also discussed in the literature [10]. Multimedia VANETs are complementary for incarcerating and sharing environmental monitoring, shadowing, traffic accidents, and disaster-based video smart city applications. Live streaming videos makes available to end-users and authorities. The motivation of this research work is the existing protocols deem the Quality of Service (QoS) of the application layer. As a result, they are tactless to the applications requirements. They also judge no intact route lifetime required to deliver the whole data, such as video and it is possibly will show the way to packet drop 
after departure of the source or destination vehicles from the route which is in progress. Considering this scenario, discovering a new route or updating the route need to be done. Therefore, there is a wide scope of inheriting further techniques in order to make the protocol appropriate for video streaming. In this research work bandwidth aware multipath geographic routing protocol for reliable video transmission is proposed.

\section{Related Works}

The current consent of the new H.265 video compression standard [11], has been intentional to restore the broadly used and renowned H.264 standard [12], gives a new-fangled prospect for real-time video transmission in decisive circumstances. This new-fangled standard, which outperforms the old one by accomplishing the same video quality with only $50 \%$ of the bit-rate [13], is probable to turn out to be a facilitating technology when trying to offer real-time video transmission in VANETs. Cuomo et al. [14] put forward the making of a vehicular Backbone Network (VBN) for elevated throughput content distribution on thoroughfare roads. In difference their work spotlighted on distributing live video streaming between vehicles. Guo et al. [13] suggested several scenarios where live video streaming between vehicles is both practicable and enviable. Soldo et al. [15] presented the Streaming Urban Video (SUV) protocol, a distributed elucidation to broadcast video streams in VANETs. The protocol recommended dividing the neighbors into four sectors and selecting one node in every sector as a candidate for rebroadcasting; even though an out of the ordinary MAC layer is necessary to sustain TDMA scheduling. Such situation prevents its implementation on real IEEE 802.11p devices.

Piñol et al. [16] proposed the primary come up to a correct simulation setting competent enough to represent real-time video transmission in VANETs. The authors presented a simulation platform and PSNR results, following the guidelines presented by Seeling and Reisslein [17], thereby offering a constructive loom on the way to simulate video transmission exactly. Wu et al. proposed distortion-aware concurrent multipath transfer (CMT-DA) solution [18]. CMT-DA examined data distribution among more than one path to minimize the video distortion based on the utility maximization theory. Wu et al. also proposed a scheduling approach that uses frame splitting based on weibull distribution and graph theory to minimize the end-to-end delivery delay and reduce out-of-order reception [19]. Xu et al. proposed a quality-aware adaptive concurrent multipath transfer solution (CMT-QA) [20]. CMT-QA takes advantages of reordering delay reduction and unnecessary fast retransmission to alleviate out-of-order data reception. Unfortunately, none of the above works considers the characteristics of the data transmitted over the path.

\section{Proposed Work}

In the proposed research work more than one path are constructed using the AOMDV routing protocol [8]. The paths within each set can share links among themselves however the two sets are said to be disjoint in order to augment reliability of the connections. It is considered that the given time is divided into fixed length time slots (e.g., $n$ second time slots) and the proposed routing mechanism iteratively manages these time slots and also probably may recalculate paths for each request depending on the measurements results gathered from the active probing of the vehicular nodes. In the proposed routing scheme, the requests are processed in sequential manner. New-fangled video transmission requests are obtained. Before processing the video transmission requests, it is first checked whether the previously accepted requests are finished so that their assigned resources are probably be released. Then in each time slot the resources are updated based on the measurement results of the vehicular nodes and the requests which their QoS demands are not fulfilled are detected and then a network allocation mechanism is called for each request. Once when the algorithm is thriving in allocating bandwidth to the request, it is admitted and the resources for that time slot is updated otherwise, the previous resource allocation does not change.

The algorithm is shown in Algorithm 1 that runs over time and given all requests, it checks whether any of the previously admitted ones is finished so that its resources can be released. Then it calls the TimeSlot algorithm to process the new requests which is portrayed in Algorithm 2. The TimeSlot algorithm first checks for the beginning of a new time slot and then checks the measurements received from different vehicular nodes to update the status of the network in terms of available bandwidth metric.

The Update Resource component takes care for recognizing the available bandwidth gathered from the active probing of the wireless vehicular nodes and will be saved in actual BW. Also along with the actual BW, min PBW (minimum predicted available bandwidth) of each link in the vehicular network is predicted by assuming $i$ is the length of the time slot. Using this prediction, it is presumed that the available bandwidth in the current time slot, $t$, which can be used for multipath provisioning of the requests arriving in $t$ is equal to the minimum of actual BW and min PBW (TimeSlot BW $[t]$ ). 


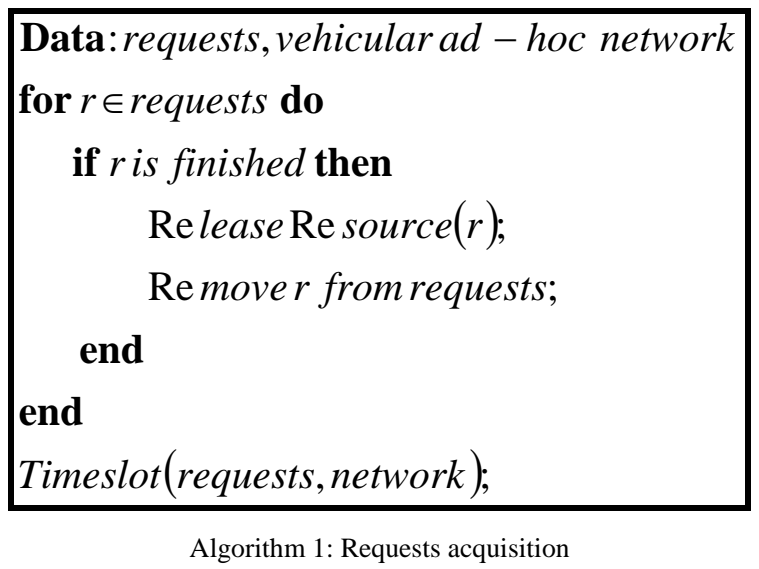

The algorithm then checks whether any of the already admitted requests are affected. The affected Request component is responsible for checking the requests and if the performance of any request is below a certain threshold it is added to the affected $R$ list to be re-configured.

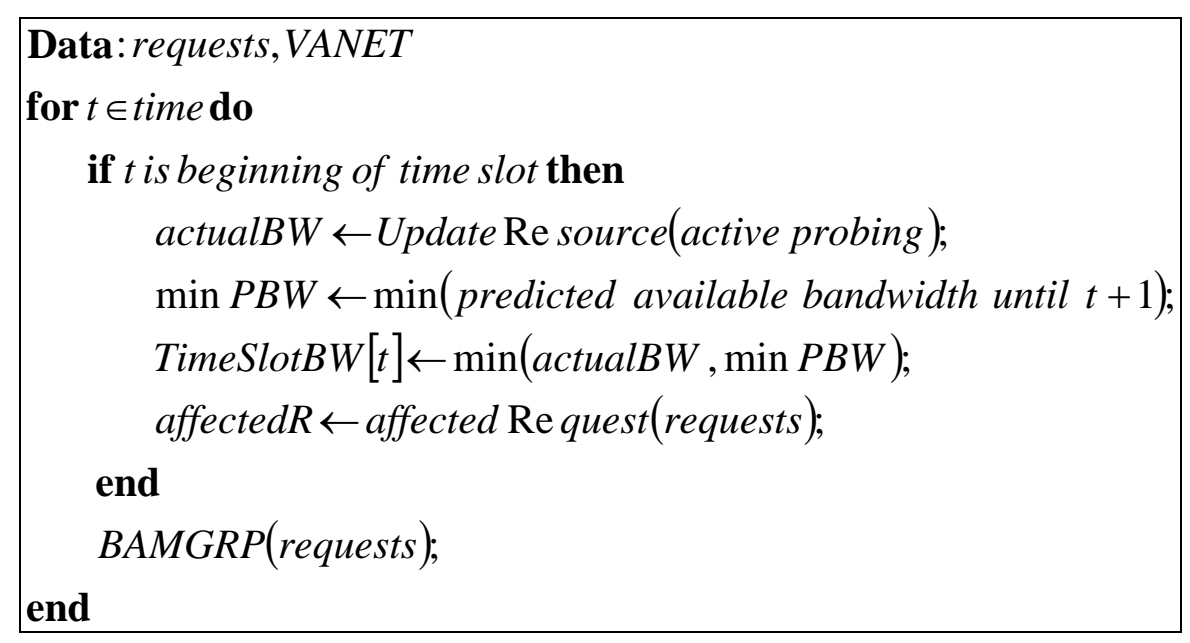

Algorithm 2: TimeSlot Algorithm

The concurrent video transmission requests are sorted based on their bandwidth demand in a descending order. For each request, the network resource usage is maintained as depicted in Algorithm 3 which is used for backtracking in case of failure.

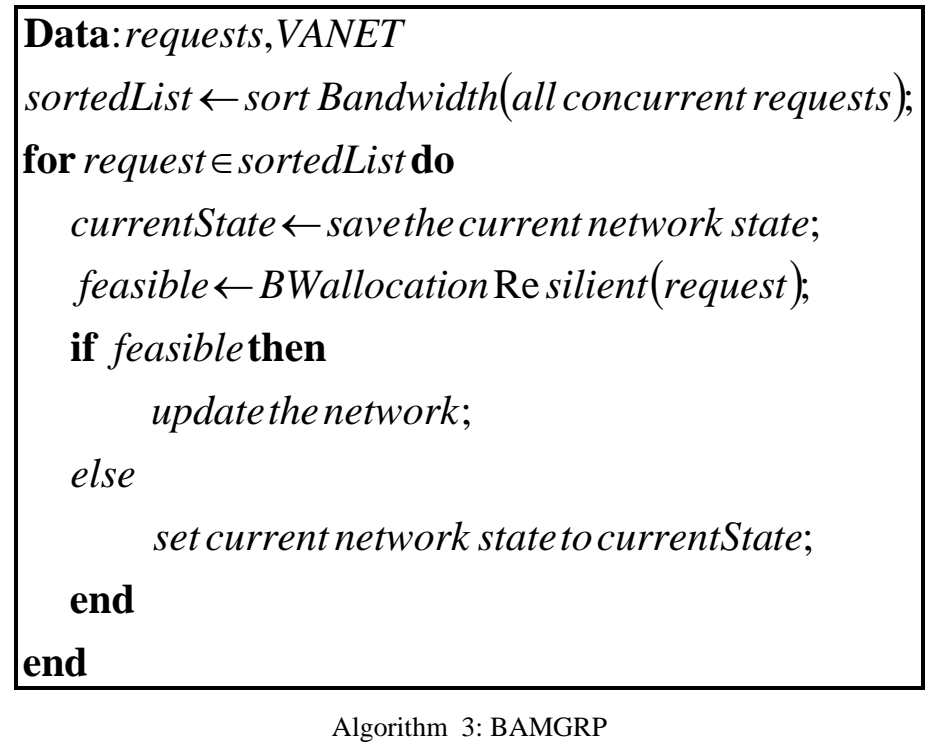

Each request is then given to the flexible bandwidth allocation algorithm which finds the close to optimal paths for each video transmission request. 


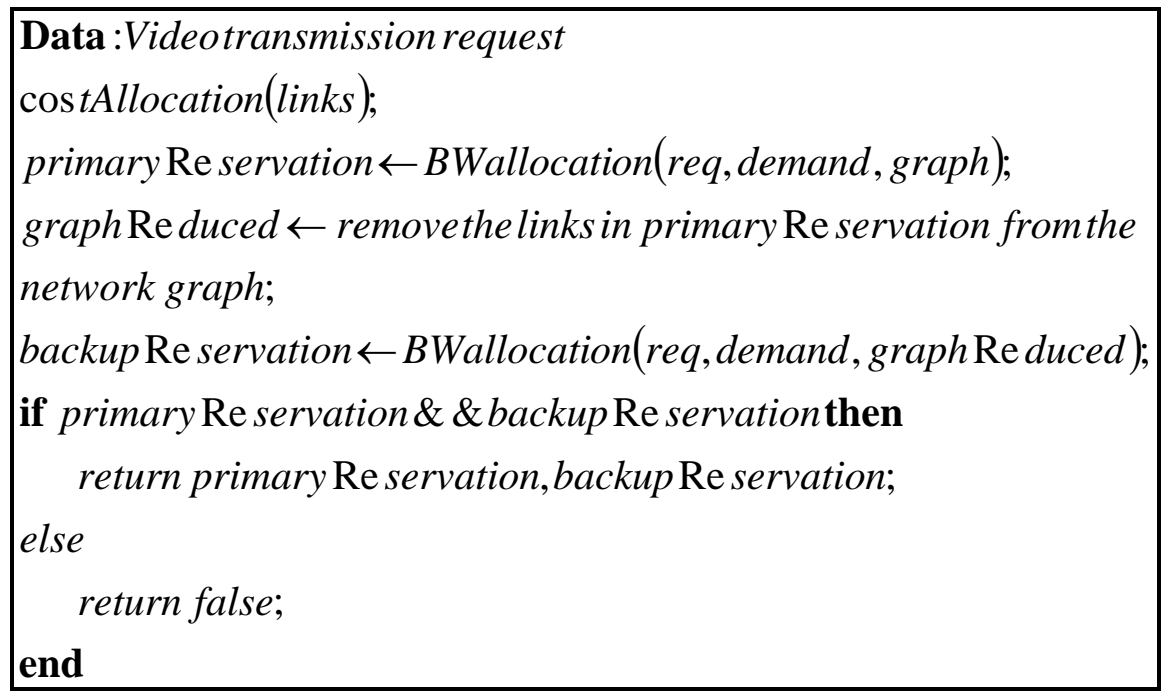

Algorithm 4: Flexible Bandwidth Allocation Algorithm

In this algorithm first the cost allocation module assigns a cost to each link of the VANET. Then the BW allocation module is called for each request for the first time to find a set of multipath as primary set. This set fulfills the request's demand in terms of bandwidth. Then the links within this set are removed from the network and the BW allocation is called for the second time on the reduced topology to find second set of multipaths as secondary/backup set. To this end, it is sufficient to only reserve bandwidth equal to the maximum bandwidth allocated on the edges of the primary paths. As such, if any link in the primary paths breaks there is enough backup bandwidth for the recovery of the affected paths. When a feasible set of primary and secondary paths are found the request is admitted and the network is updated according to the new allocations otherwise the request is rejected. By choosing the paths with more available bandwidth, the algorithm first tries to select a single path for each set and only if there is not enough capacity in a single path to fulfill the requests demand, it iterates to find multipaths. The reason is that in video streaming, it is preferable to lessen the traffic splitting so that the packet re-ordering (out of order packet problem) is less challenging in the edge points.

\section{Simulation Settings, Results and Discussions}

Simulation settings are presented in Table 1 . The performance metrics such as route length, packet end-to-end delay, freezing delay, number of delivered packets and packet loss ratio are taken into account. The proposed BAMGRP is compared with AMGR protocol. The network region consisting of 1000 vehicular nodes are presented in Fig.1. As per the network topology, when the route length is increased the quality of video transmission also increases. From the Fig.2 it is evident that the proposed BAMGRP has better route length than that of AMGR protocol. It is noteworthy that when the end-to-end delay is reduced it results in better video transmission speed. Fig. 3 portrays the results of simulation time versus packet end-to-end delay. From the obtained results it shows that the proposed BAMGRP acquires less packet end-to-end delay than that of AMGR protocol [9]. Freezing delay also taken into account in order to witness the quality of video transmission. Less freezing delay results in better video quality. Fig.4 presents the simulation results in terms of simulation time versus freezing delay. From the results it is obvious that the proposed BAMGRP consumes lesser freezing delay when compared to AMGR protocol.

Throughput or number of packets delivered is the measure used to evaluate the performance of the protocol by which the total number of successful packets reached towards the destination. Fig.5 shows the performance comparison of the protocols in terms of simulation time versus number o delivered packets. It is evident that the proposed BAMGRP attains better throughput than that of AMGR protocol. Fig.6 presents average packet loss ratio of the protocols and from the results it can be perceived that the proposed BAMGRP has less packet loss ratio that ensures better video transmission. The result values are presented from Table 2 to Table 6 . 
TABLE I Simulation Settings

\begin{tabular}{|l|l|}
\multicolumn{1}{c|}{ Parameters } & \multicolumn{1}{c|}{ Values } \\
\hline Simulation area & $2.5 \mathrm{~km} \mathrm{X} 3.5 \mathrm{~km}$ \\
\hline Number of nodes & 1000 \\
\hline Number of intersections & 250 \\
\hline Number of streets & 513 \\
\hline Vehicle speed & $3-13$ meter/second \\
\hline Transmission range & 250 meters \\
\hline Minimum data rate & 6 Mbps \\
\hline Simulation time & 12 seconds \\
\hline Beacon interval & 5 seconds \\
\hline
\end{tabular}



Fig.1. Network Setup

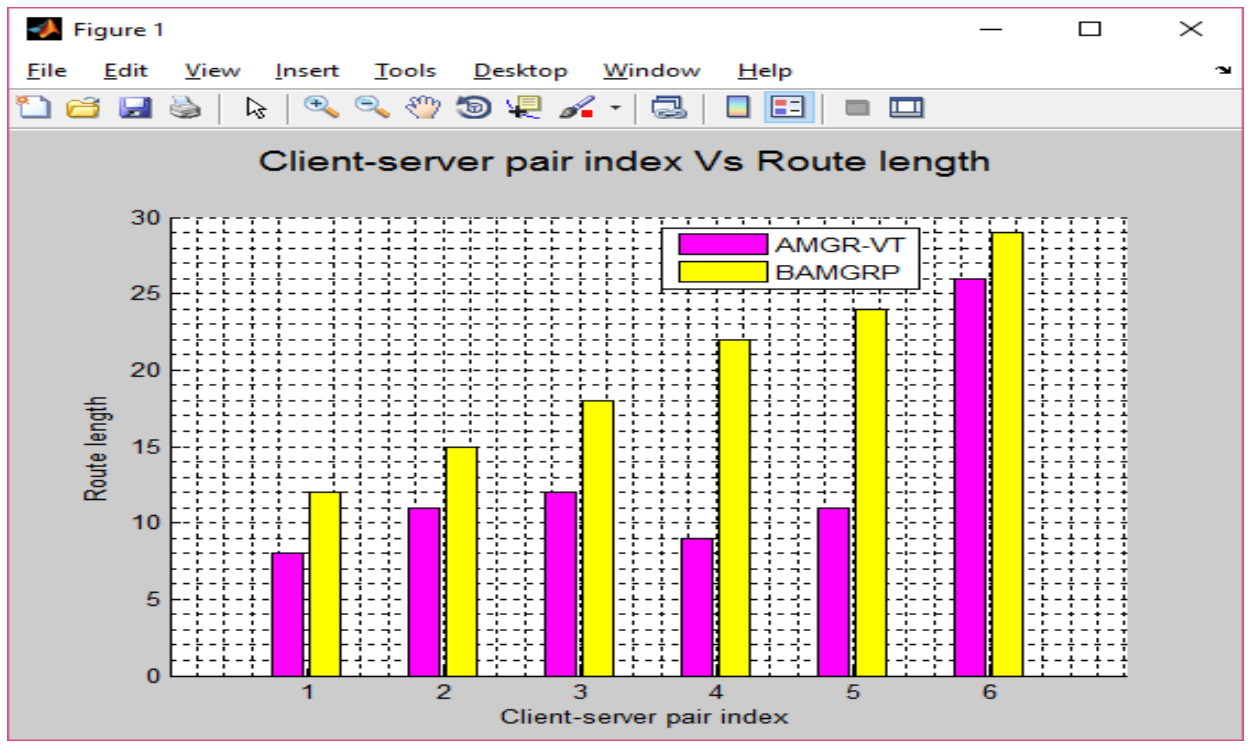

Fig. 2. Client - Server Pair Index Vs Route Length 


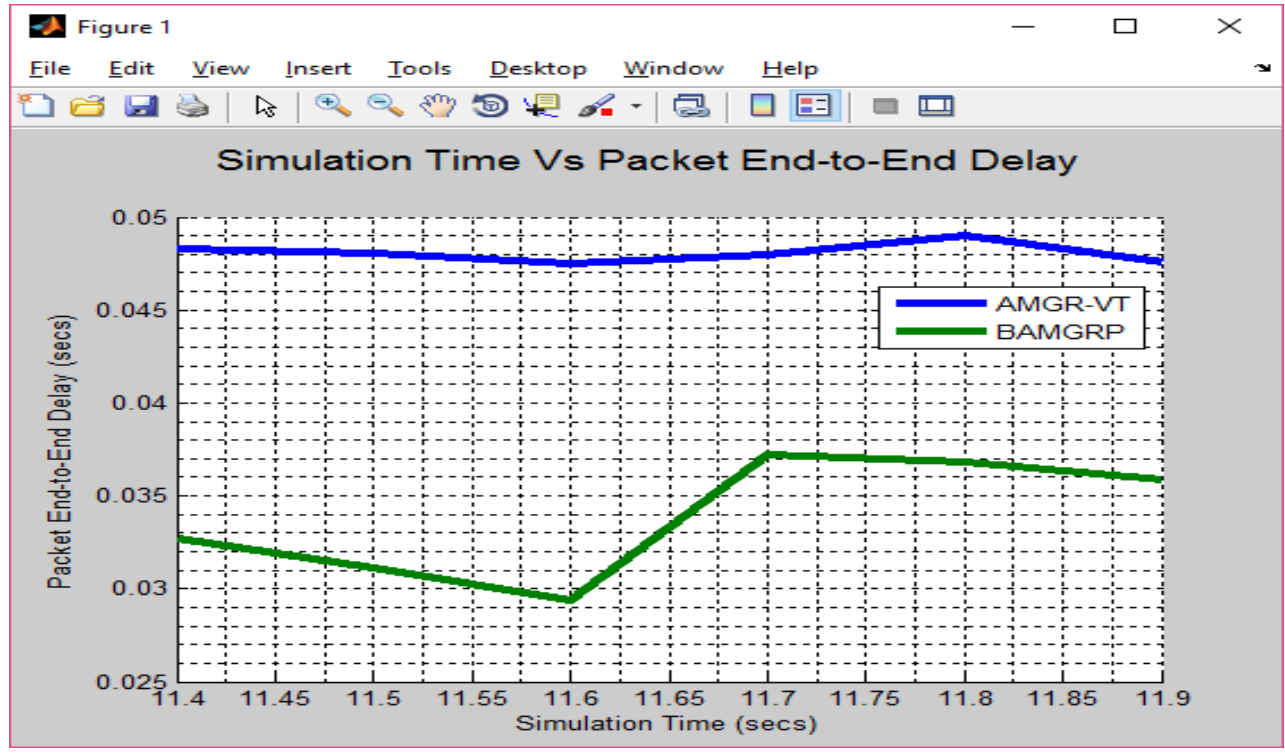

Fig. 3. Simulation Time Vs Packet End - to - End Delay

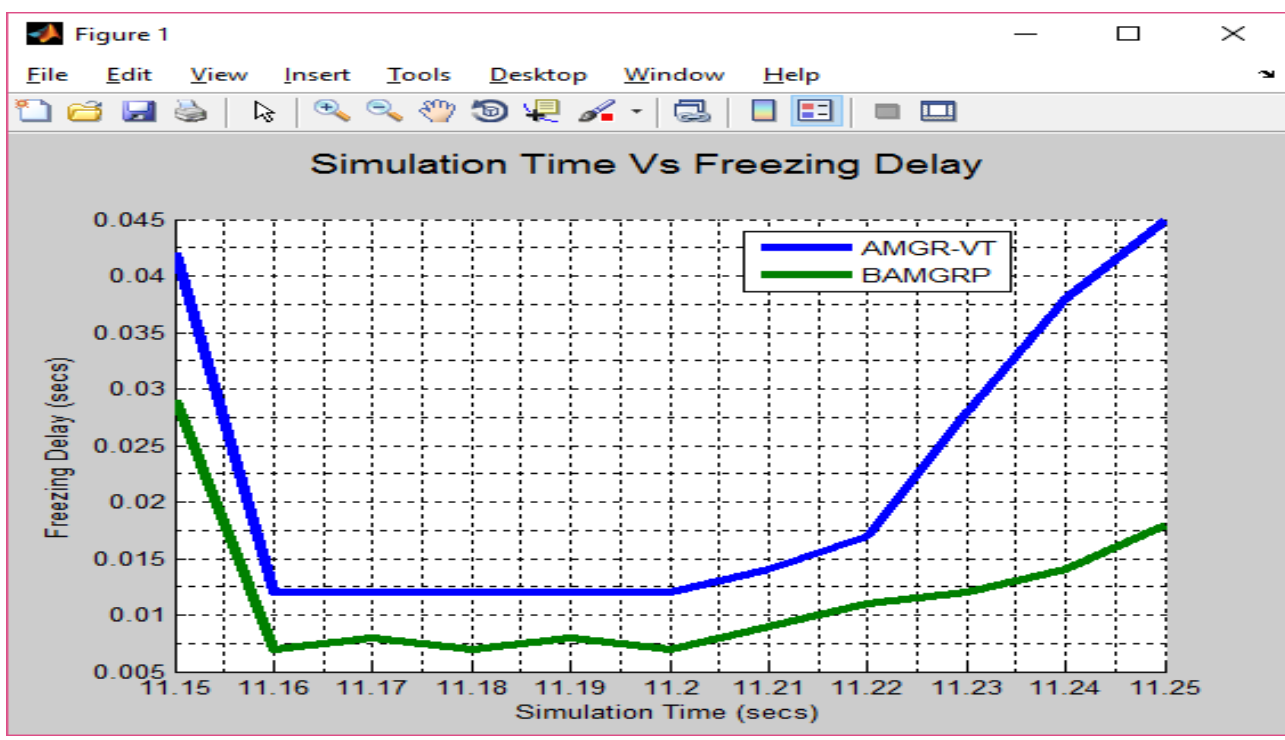

Fig. 4. Simulation Time Vs Freezing Delay

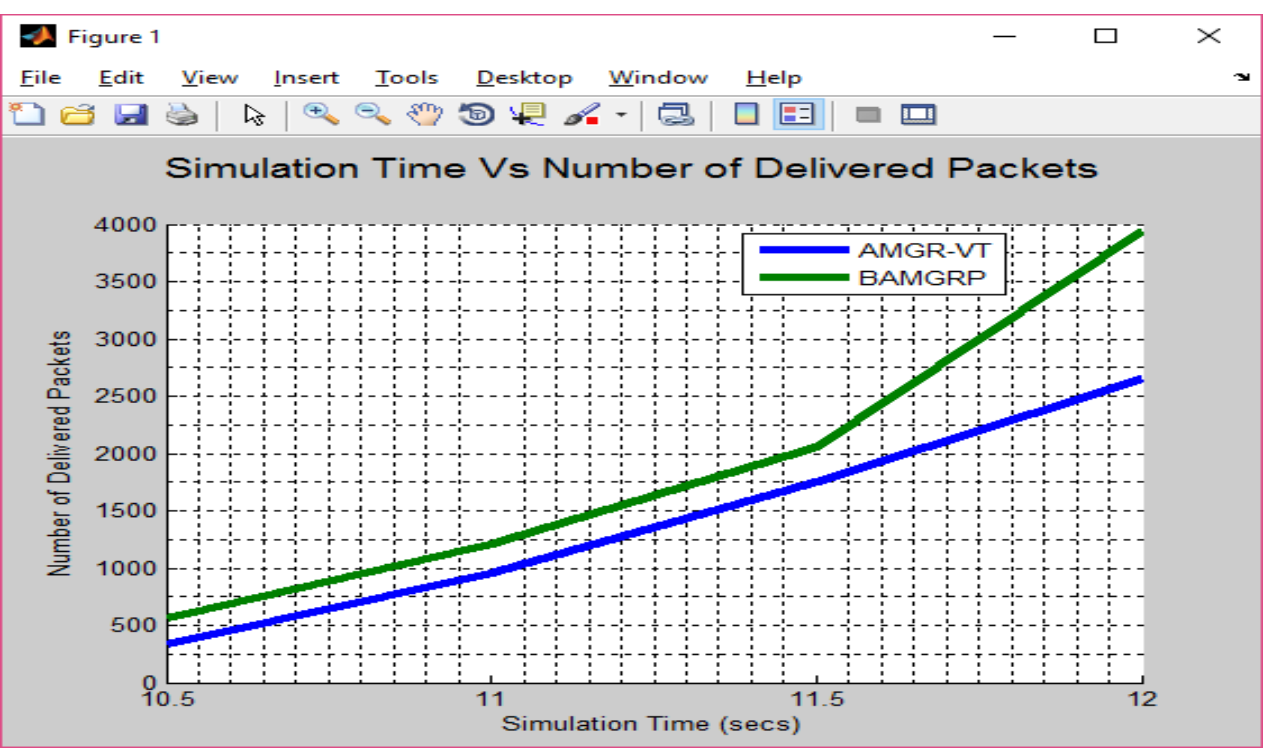

Fig.5. Simulation Time Vs Number of Delivered Packets 


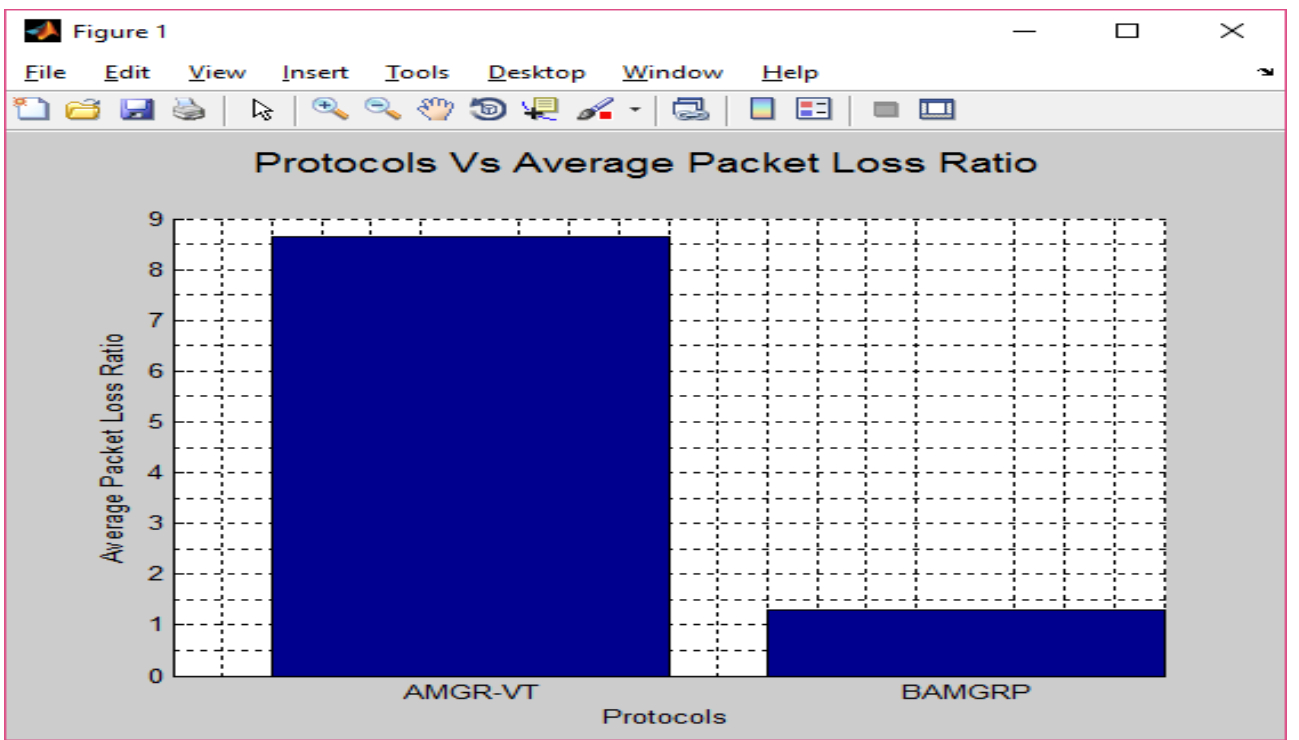

Fig. 6. Protocols Vs Average Packet Loss Ratio

TABLE II Client - Server Pair Index Vs Route Length

\begin{tabular}{|c|c|c|}
\hline & \multicolumn{2}{|c|}{ Route Length (count) } \\
\hline Pair Index & AMGR-VT & BAMGRP \\
\hline 1 & 8 & 12 \\
\hline 2 & 11 & 15 \\
\hline 3 & 12 & 18 \\
\hline 4 & 9 & 22 \\
\hline 5 & 11 & 24 \\
\hline 6 & 26 & 29 \\
\hline
\end{tabular}

TABLE III . Simulation Time Vs Packet End-to-End Delay

\begin{tabular}{|c|c|c|}
\hline & \multicolumn{2}{|c|}{ Packet End-to-End Delay (seconds) } \\
\hline Simulation Time (seconds) & AMGR-VT & BAMGRP \\
\hline 11.4 & 0.0483 & 0.0327 \\
\hline 11.5 & 0.0481 & 0.0311 \\
\hline 11.6 & 0.0475 & 0.0294 \\
\hline 11.7 & 0.048 & 0.0372 \\
\hline 11.8 & 0.049 & 0.0368 \\
\hline 11.9 & 0.0476 & 0.0359 \\
\hline
\end{tabular}


TABLE IV Simulation Time Vs Freezing Delay

\begin{tabular}{|c|c|c|}
\hline & \multicolumn{2}{|c|}{ Freezing Delay (seconds) } \\
\hline Simulation Time (seconds) & AMGR-VT & BAMGRP \\
\hline 11.15 & 0.042 & 0.029 \\
\hline 11.16 & 0.012 & 0.007 \\
\hline 11.17 & 0.012 & 0.008 \\
\hline 11.18 & 0.012 & 0.007 \\
\hline 11.19 & 0.012 & 0.008 \\
\hline 11.2 & 0.012 & 0.007 \\
\hline 11.21 & 0.014 & 0.009 \\
\hline 11.22 & 0.017 & 0.011 \\
\hline 11.23 & 0.028 & 0.012 \\
\hline 11.24 & 0.038 & 0.014 \\
\hline 11.25 & 0.045 & 0.018 \\
\hline
\end{tabular}

TABLE V Simulation Time Vs Number of Packets Delivered

\begin{tabular}{|c|c|c|}
\hline & \multicolumn{2}{|c|}{ Number of Packets Delivered (packets) } \\
\hline Simulation Time (seconds) & AMGR-VT & BAMGRP \\
\hline 10.5 & 332 & 563 \\
\hline 11 & 958 & 1204 \\
\hline 11.5 & 1759 & 2062 \\
\hline 12 & 2658 & 3937 \\
\hline
\end{tabular}

TABLE VI Packet Loss Ration of Protocols

\begin{tabular}{|c|c|}
\hline \multicolumn{2}{|c|}{ Packet Loss Ratio (count) } \\
\hline AMGR-VT & BAMGRP \\
\hline 8.65 & 1.31 \\
\hline
\end{tabular}

\section{Conclusion and Future Works}

Vehicular ad hoc network shortly termed as VANET is the application of mobile ad hoc network. Video transmission is one of the challenging research problems in the field of VANET. This research work aims in design and development of bandwidth aware multipath geographic routing protocol for reliable video transmission in VANETs. The proposed BAMGRP uses multipath routing strategy that inherits features of AOMDV routing protocol for multiple paths discovery. Bandwidth aware routing is used and the vehicular nodes are allowed to transmit video data over the network. Simulations are performed using MATLAB. 1000 nodes are deployed in the simulation region and the performance metrics such as route length, packet end-to-end delay, freezing delay, number of delivered packets and packet loss ratio. Results are compared with

\section{References}

[1] M. K. Jiau, S. C. Huang, J. N. Hwang and A. V. Vasilakos, "Multimedia Services in Cloud-Based Vehicular Networks," in IEEE Intelligent Transportation Systems Magazine, vol. 7, no. 3, pp. 62-79, 2015.

[2] M. A. Al-Maqri, M. Othman, B. Mohd Ali, Z. M. Hanapi, "Packet-based Polling Scheme for Video Transmission in IEEE 802.11e WLANs," in Procedia Computer Science, vol. 83, pp. 337-344, 2016.

[3] M. Gerla, C. Wu, G. Pau, X. Zhu, "Content Distribution in VANETs," in Vehicular Communications, vol. 1, no. 1, pp. 3 - $12,2014$.

[4] R. F. Atallah, M. J. Khabbaz, C. M. Assi, "Vehicular networking: A survey on spectrum access technologies and persisting challenges," in Vehicular Communications, vol. 2, no. 3, pp. 125 - 149, 2015.

[5] R. Jurdak, C. V. Lopes and P. Baldi, "A survey, classification and comparative analysis of medium access control protocols for ad hoc networks," in IEEE Communications Surveys \& Tutorials, vol. 6, no. 1, pp. 2-16, 2004.

[6] L. Junhai, Y. Danxia, X. Liu and F. Mingyu, "A Survey of Multicast Routing Protocols For Mobile Ad-hoc Networks," in IEEE Communications Surveys \& Tutorials, vol. 11, no. 1, pp. 78-91, 2009.

[7] B. T. Sharef, R. A. Alsaqour, M. Ismail, "Vehicular Communication Ad-hoc Routing Protocols: A Survey," in Journal of Network and Computer Applications, vol. 40, no. 2, pp.363-396, 2014.

[8] A. Vidwans, A. K. Shrivastava and M. Manoria, "QoS Enhancement of AOMDV Routing Protocol Using Queue Length Improvement," 2014 Fourth International Conference on Communication Systems and Network Technologies, Bhopal, 2014, pp. 275278.

[9] M. Asgharpoor Salkuyeh and B. Abolhassani, "An Adaptive Multipath Geographic Routing for Video Transmission in Urban VANETs," in IEEE Transactions on Intelligent Transportation Systems, vol. 17, no. 10, pp. 2822-2831, Oct. 2016. 
[10] S. S. Baboo and B. Narasimhan, "An Energy-Efficient Congestion-Aware Routing Protocol for Heterogeneous Mobile Ad Hoc Networks," 2009 International Conference on Advances in Computing, Control, and Telecommunication Technologies, Trivandrum, Kerala, 2009, pp. 344-350.

[11] H.265 standard <http://www.itu.int/rec/T-REC-H.265>.

[12] H.264 standard <http://www.itu.int/rec/T-REC-H.264>.

[13] H. Koumaras, M. Kourtis, D. Martakos, Benchmarking the encoding efficiency of h.265/hevc and h.264/avc, in: Future Network Mobile Summit (FutureNetw), 2012, 2012, pp. 1-7.

[14] F. Cuomo, I. Rubin, A. Baiocchi, P. Salvo, Enhanced VANET broadcast throughput capacity via a dynamic backbone architecture, Ad Hoc Networks 21 (2014) 42-59.

[15] F. Soldo, C. Casetti, C. Chiasserini, P. Chaparro, Video streaming distribution in vanets, IEEE Trans. Parallel Distrib. Syst. 22 (7) (2011) 1085-1091.

[16] P. Pin' ol, O. López, M. Martínez, J. Oliver, M.P. Malumbres, Modeling video streaming over vanets, in: Proceedings of the 7th ACM Workshop on Performance Monitoring and Measurement of Heterogeneous Wireless and Wired Networks, PM2HW2N'12, ACM, New York, NY, USA, 2012, pp. 7-14.

[17] P. Seeling, M. Reisslein, Video transport evaluation with h.264 video traces, Commun. Surv. Tut., IEEE 14 (4) (2012) 1142-1165.

[18] J. Wu, B. Cheng, C. Yuen, Y. Shang, J. Chen, Distortion-aware concurrent multipath transfer for mobile video streaming in heterogeneous wireless networks, IEEE Trans. Mob. Comput. 14 (4) (2015) 688-701.

[19] J. Wu, B. Cheng, Y. Shang, J. Huang, J. Chen, A novel scheduling approach to concurrent multipath transmission of high definition video in overlay networks, J. Netw. Comput. Appl. 44 (2014) 17-29.

[20] C. Xu, T. Liu, J. Guan, H. Zhang, G.-M. Muntean, CMT-QA: quality-aware adaptive concurrent multipath data transfer in heterogeneous wireless networks, IEEE Trans. Mob. Comput. 12 (11) (2013) 2193-2205. 